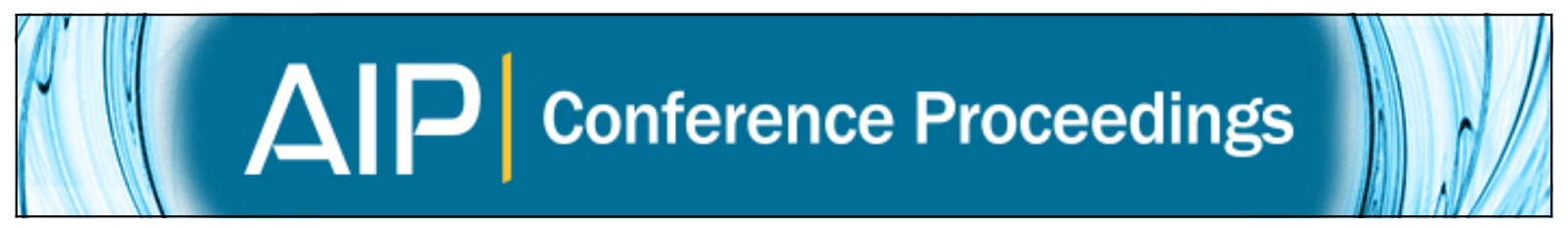

\title{
Ultra-precise Masses and Magnitudes for the Gliese 268 M-dwarf Binary
}

R. K. Barry, B. O. Demory, D. Ségransan, T. Forveille, W. C. Danchi, E. Di Folco, D. Queloz, G. Torres, W. A. Traub, X. Delfosse, M. Mayor, C. Perrier, and S. Udry

Citation: AIP Conference Proceedings 1094, 780 (2009); doi: 10.1063/1.3099232

View online: http://dx.doi.org/10.1063/1.3099232

View Table of Contents: http://scitation.aip.org/content/aip/proceeding/aipcp/1094?ver=pdfcov

Published by the AIP Publishing

Articles you may be interested in

The AstraLux large M dwarf survey

AIP Conf. Proc. 1094, 935 (2009); 10.1063/1.3099272

Dwarf Archives: A Compendium of M, L, and T Dwarf Data

AIP Conf. Proc. 1094, 924 (2009); 10.1063/1.3099269

Decametric observations of active M-dwarfs

AIP Conf. Proc. 1094, 680 (2009); 10.1063/1.3099205

Fundamental Properties of Low-Mass Stars and Brown Dwarfs

AIP Conf. Proc. 1094, 258 (2009); 10.1063/1.3099100

High Precision Dynamical Masses for Brown Dwarf Binaries

AIP Conf. Proc. 1094, 112 (2009); 10.1063/1.3099079 


\title{
Ultra-precise Masses and Magnitudes for the Gliese 268 M-dwarf Binary
}

\author{
R. K. Barry*, B. O. Demory ${ }^{\dagger}$, D. Ségransan ${ }^{\dagger}$, T. Forveille**, W. C. Danchi*, \\ E. Di Folco ${ }^{\dagger}$, D. Queloz ${ }^{\dagger}$, G. Torres ${ }^{\ddagger}$, W. A. Traub ${ }^{\S}$, X. Delfosse ${ }^{\dagger}$, \\ M. Mayor ${ }^{\dagger}$, C. Perrier** and S. Udry ${ }^{\dagger}$ \\ *NASA Goddard Space Flight Center, Exoplanets and Stellar Astrophysics, Greenbelt, MD 20771 \\ $\dagger$ Observatoire Astronomique de l'Université de Genève, 51 ch des Maillettes, \\ Sauverny, CH-1290, Versoix, Switzerland \\ ** Observatoire de Grenoble, 414 rue de la Piscine, Domaine Universitaire de St Martin d'Héres, \\ 38041 Grenoble, France \\ ${ }^{\ddagger}$ Harvard-Smithsonian Center for Astrophysics, 60 Garden St., Cambridge, MA 02138 USA \\ ${ }^{\S}$ California Institute of Technology, Jet Propulsion Laboratory, Pasadena, CA 91125 USA
}

\begin{abstract}
Recent advances in astrometry using interferometry and precision radial velocity techniques combined allow for a significant improvement in the precision of masses of M-dwarf stars in visual systems. We report recent astrometric observations of Gliese 268, an M-dwarf binary with a 10.4 day orbital period, with the IOTA interferometer and radial velocity observations with the ELODIE instrument. Combining these measurements leads to preliminary masses of the constituent stars with uncertainties of $0.4 \%$. The masses of the components are $0.22596 \pm 0.00084 M_{\odot}$ for the primary and $0.19230 \pm 0.00071 M_{\odot}$ for the secondary. The system parallax is determined by these observations to be $0.1560 \pm .0030$ arcsec $(2.0 \%$ uncertainty) and is within Hipparcos error bars $(0.1572 \pm .0033)$. We tested these physical parameters, along with the near-infrared luminosities of the stars, against stellar evolution models for low-mass stars. Discrepancies between the measured and theoretical values point toward a low-level departure from the predictions. These results are among the most precise masses measured for visual binaries.
\end{abstract}

Keywords: Low Mass Stars, Binary Stars, Normal Stars, Luminosity, Mass Function

PACS: 95.10.Ce:95.10.Eg:97.10.-q:97.10.Nf:97.10.Xq:97.20.Jg: 97.80.Af:97.10.Vm

\section{INTRODUCTION AND OBSERVATIONS}

M dwarf stars are distinctly underrepresented in studies that have made precise determinations of stellar mass. As a result, it is important to find additional systems in this mass regime for which masses and luminosities are accurately determined to constrain models. One candidate system, the double-lined spectroscopic M dwarf binary, G1 268 (HIP34603, $\left.\alpha=7^{h} 10^{m} 01.83^{s}, \delta=38^{\circ} 31^{\prime} 45.1^{\prime \prime}, J 2000.0\right)$, is an BY Dra binary consisting of late-type cool stars with narrow absorption lines $\left(V \sin (i)=2 \mathrm{~km} \mathrm{~s}^{-1}\right)$.

We conducted extensive observations of G1268 at the Observatoire de Haute Provence, Saint Michel l'Observatoire, France using the ELODIE instrument on the $1.93 \mathrm{~m}$. telescope. This fiber-fed echelle spectrograph in a single exposure can record a spectrum at a resolution of $R=42,000$ over a bandpass from $3906 \AA$ to $6811 \AA$ on a $1024 \times 1024$ CCD [5]. The accuracy and efficiency of the instrument together with its long-term stability admit the measurement of radial velocities with an accuracy of better than $15 \mathrm{~m} \mathrm{~s}^{-1}$ for stars up to $9^{\text {th }}$ magnitude in $\mathrm{V}$ while it is also capable of measuring 
TABLE 1. A listing of the derived orbital and binary parameters of G1 268. We give the corresponding results from the work of Tomkin \& Pettersen [3] when available to show the improvements obtained through our new observations.

\begin{tabular}{ccc}
\hline Parameter & Tomkin \& Pettersen (1986) & Best Fit Value \\
\hline Period (days) & $10.428 \pm 0.002$ & $10.426753 \pm 0.000013$ \\
$T_{o}$ (BJD - 2400000) & $45770.8 \pm 0.09$ & $50483.5501 \pm 0.0018$ \\
Eccentricity & $0.34 \pm 0.02$ & $0.32077 \pm 0.00036$ \\
$\omega(\mathrm{deg})$ & $217.7 \pm 3.9$ & $211.922 \pm 0.067$ \\
$\Omega(\mathrm{deg})$ & - & $89.57 \pm 0.96$ \\
$i(\mathrm{deg})$ & - & $100.42 \pm 0.37$ \\
a(mas) & - & $10.90 \pm 0.21$ \\
$K_{1}\left(k m s^{-1}\right)$ & $32.5 \pm 0.7$ & $34.795 \pm 0.013$ \\
$K_{2}\left(k m s^{-1}\right)$ & $39.3 \pm 0.5$ & $40.886 \pm 0.018$ \\
$M_{1}\left(M_{\odot}\right)$ & - & $0.22596 \pm 0.00084$ \\
$M_{2}\left(M_{\odot}\right)$ & - & $0.19230 \pm 0.00071$ \\
H-band flux fraction* & - & $0.1560 \pm 0.0030$ \\
Parallax (arcsec) ${ }^{\dagger}$ & - & \\
\hline H-band flux fraction given as $\frac{\text { flux } 1}{\text { flux } 1+\text { flux }^{*}}$ & \\
${ }^{\dagger}$ Hipparcos parallax is $0.1572 ” \pm 0.0033^{\prime \prime}$ &
\end{tabular}

velocities to about $1 \mathrm{~km} \mathrm{~s}^{-1}$ for stars up to $16^{\text {th }}$ magnitude. Data were immediately processed with a system integrated with the spectrograph control software, which produces optimally extracted and wavelength calibrated spectra. These algorithms and processes are thoroughly described in Baranne et al. [5]. Spectra were obtained on 55 nights using appropriate calibrator stars to remove telluric features. The spectra were cross-correlated against a template derived by Delfosse et al. [6] from a high S/N spectrum of G1699 (Barnard's star, M4.0 V) [7]. Subsequently, a double Gaussian is fitted to the twin-peaked cross-correlation function and the centroid of each feature was used to determine the orbital velocity of the component stars for each particular observation.

We also observed Gl 268 using the Infrared and Optical Telescope Array (IOTA) at the F. L. Whipple Observatory (Arizona) over a period of six months from October, 2005 to April, 2006. IOTA has baselines between 5 - $38 \mathrm{~m}$ and fiber-fed IONIC3 combiner optics [8] which we used to measure 3 visibilities $\left(V^{2}\right)$ and 1 closure phase (CP) simultaneously in the broadband $\mathrm{H}$ band filter $(\lambda=1.65 \mu \mathrm{m}, \Delta \lambda=0.3 \mu \mathrm{m})$. Data analysis procedures have been documented in recent papers [9]. For the data presented here, we have adopted a calibration error of $3 \%$.

\section{DATA ANALYSIS AND ORBITAL SOLUTION}

The combined orbit fit was determined by using ORBIT originally written by $\mathrm{A}$. Tokovinin then improved by T. Forveille [11]. It performs a least squares fit to all available radial-velocity and interferometric $V^{2}$ measurements, with weights inversely proportional to the square of their standard errors. Instead of fitting individual velocities, we use the correlation profile in its entirety which improves the robustness of the resulting 
orbital solution. We corrected those profiles by estimating the difference between the actual profile for each component and a Gaussian profile by averaging recentred profiles with the other component subtracted. Then, those corrections are subtracted from the profile on the next iteration. This mitigates systematics and removes potential anticorrelations. Moreover, the narrowness of spectral lines allows an increased sampling of independent points in residuals obtained after profile-to-profile subtraction, reducing further potential bias contribution. Adding interferometric $V^{2}$ visibilities leaves spectroscopically determined parameters unchanged but strongly constrains the inclination angle $(i)$ that may be used to derive actual masses, as well as the semi-major axis $(a)$ and the longitude of ascending node $(\Omega)$. Since G1 268 system was not resolved on the smallest baseline (BC), at first we included only the longest baseline (AB) $V^{2}$ measurements in the fit. Unfortunately, lack of spatial frequency coverage did not allow for a unique solution in the parameter space. A $\chi^{2}$ map revealed several equivalent minima and the $\chi^{2}$ clearly showed a strong degeneracy between $\Omega$ and inclination angle, $i$. Although the BC baseline $V^{2}$ measurements were not used here, inclusion of AC baseline data allowed us to remove all but one orbital solution without changing the reduced $\chi^{2}$. The resulting better sampling of $(\mathrm{u}, \mathrm{v})$ plane improved the precision of the derived orbital parameters. Statistics from each data source yield a $\chi^{2}$ consistent with respective number of measurements. However, due to the lack of interferometric data at both ends of orbit ellipsoid, the error corresponding to the parallax determined within the combined fit is only slightly more precise than that of Hipparcos but is still strongly correlated, being discrepant by only $0.1 \sigma$. The derived parameters and associated standard errors are given in Table 1. The latter were computed by using the full covariance matrix of the resulting orbital global adjustement.

\section{CONCLUSION}

A comparison with evolutionary models from Baraffe et al. [12] indicated that the best fit corresponds to a mixing length of $1 H_{p}$, and $Y=0.275$ helium abundance, a $[\mathrm{Fe} / \mathrm{H}]=0$ metallicity [13] and an age of 10 Gyr. Shown on Fig. 11 is an H-band massmagnitude diagram with both components plotted with models corresponding to the best fit (solid), 7.9 Gyr age (dash) and $Y=0.25 ; 10 \mathrm{Gyr}$ (dot). Although discrepancies are of the order of $2 \sigma$, we note that the observed lower mass-magnitude slope can't be reproduced by changing helium abundance or convection efficiency (mixing length). Changing age is not relevant for $\mathrm{M}$ dwarfs such as $\mathrm{Gl} 268$, as they typically exhibit negligible luminosity variation once on the main-sequence. We note that parallax error would tend to move the data points horizontally and together so any discrepancy of the combined two points from the model is significant. Although activity effects are barely

noticeable in $\mathrm{H}$ band for this range of masses as discussed above, Gl 268's activity should be low. Additionally, the orbital period is sufficiently long to avoid tidal locking effects that would have induced an activity contribution observed in close systems [2]. 


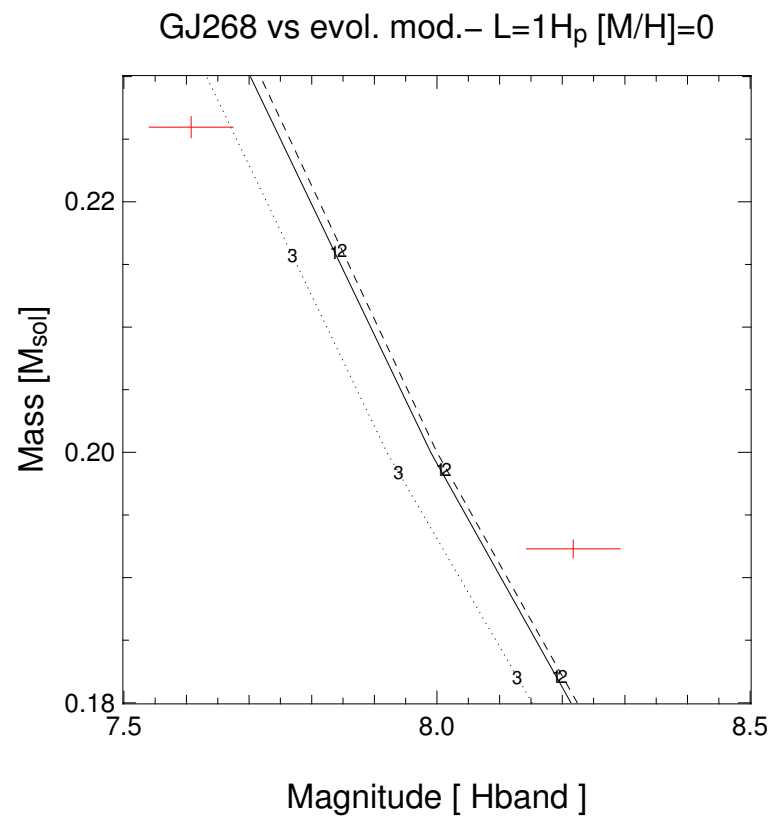

FIGURE 1. Derived physical orbit parameters (red data points) of the M dwarf binary Gl268 compared to the prediction of a late-type stellar model [12]. (1, solid) Best fit for both stars, mixing length of $1 \mathrm{Hp}$, $Y=0.275,[\mathrm{Fe} / \mathrm{H}]=0,10 \mathrm{Gyr}$. (2, dash) Mixing length of $1 \mathrm{Hp}, Y=0.275,[\mathrm{Fe} / \mathrm{H}]=0,7.9$ Gyr. (3, dot) Mixing length of $1 \mathrm{Hp}, Y=0.25,[\mathrm{Fe} / \mathrm{H}]=0,10 \mathrm{Gyr}$

\section{ACKNOWLEDGMENT}

We are grateful to the National Aeronautics and Space Administration, the University of Grenoble Observatory, Jet Propulsion Laboratory, the California Association for Research in Astronomy, and to the Harvard-Smithsonian Center for Astrophysics for support of this research. We are also grateful for the work on software to reduce the interferometric data from IOTA by J. Monnier. BOD acknowledges the support of the Fonds National Suisse de la Recherche Scientifique. GT acknowledges partial support from NSF grant AST-0708229

\section{REFERENCES}

1. Torres, G., \& Ribas, I., 2002, Astrophysical Journal, 567, 1140

2. Chabrier, G., \& Baraffe, I., 2007, Astronomy and Astrophysics,327,1039

3. Tomkin, J., \& Pettersen, B., 1986, Astronomical Journal, 92, 1424

4. Perryman, M., Lindegren, L., et al., 1997, Astronomy and Astrophysics, 323, L49

5. Baranne, A., Queloz, D., et al., 1996, Astronomy and Astrophysics Supplements, 119, 373

6. Delfosse, X., Forveille, T., et al., 1999, Astronomy and Astrophysics, 344,897

7. Queloz, D., 1995, PhD Thesis

8. Berger, J., Haguenauer, P., et al., 2003, Proc. SPIE,4838

9. Monnier, J., Traub, W., et al., 2004, Astrophysical Journal Letters,602,L57

10. Andersen, J., 1991, Astronomy and Astrophysics Review,3,91

11. Forveille, T., Beuzit, J., et al., 1999, Astronomy and Astrophysics,351,619

12. Baraffe, I., Chabrier, G., et al., 1998, Astronomy and Astrophysics,337,403

13. Bonfils, X., Delfosse, X., et al., 2005, Astronomy and Astrophysics, 442, 635 